\title{
Absolute Lymphocyte Count Changes During Neoadjuvant Chemotherapy are Associated with Prognosis of HER2-Positive Breast Cancer Patients
}

Naoki Miyamoto ( $\square$ miyamoto.naoki@tokushima-u.ac.jp )

Tokushima Daigaku Igakubu Daigakuin Ikgaku Kyoikubu

Hiroaki Inoue

Tokushima Daigaku Igakubu Daigakuin Ikgaku Kyoikubu https://orcid.org/0000-0003-0043-942X

Tomohiro Inui

Tokushima Daigaku Igakubu Daigakuin Ikgaku Kyoikubu

\section{Soichiro Sasa}

Tokushima Daigaku Igakubu Daigakuin Ikgaku Kyoikubu

\section{Mariko Aoyama}

Tokushima Daigaku Igakubu Daigakuin Ikgaku Kyoikubu

\section{Kazumasa Okumura}

Tokushima Daigaku Igakubu Daigakuin Ikgaku Kyoikubu

Hiroaki Toba

Tokushima Daigaku Igakubu Daigakuin Ikgaku Kyoikubu

Hiromitsu Takizawa

Tokushima Daigaku Igakubu Daigakuin Ikgaku Kyoikubu

Akira Tangoku

Tokushima Daigaku Igakubu Daigakuin Ikgaku Kyoikubu

\section{Research Article}

Keywords: HER2 breast cancer, neoadjuvant chemotherapy, lymphocyte counts, trastuzumab, cellmediated cytotoxicity

Posted Date: March 16th, 2021

DOI: https://doi.org/10.21203/rs.3.rs-310226/v1

License: (1) (1) This work is licensed under a Creative Commons Attribution 4.0 International License. Read Full License 


\section{Abstract}

Purpose: To investigate the relation of absolute lymphocyte count (ALC) changes during neoadjuvant chemotherapy for human epidermal growth factor receptor-2 (HER2)-positive breast cancer patients and their prognosis.

Methods: From January 2010 to December 2019, patients diagnosed with HER2-positive breast cancer and treated with trastuzumab-based neoadjuvant chemotherapy (NAC) were included in this retrospective cohort study. The ALC ratio was Blood cell count estimates before and after NAC were evaluated to calculate the ALC ratio. The optimal cut-off for the ALC ratio was identified using the receiver operating characteristic (ROC) curve analysis and Youden's index. The relationship between the ALC ratio and disease-free survival (DFS) was measured using the Kaplan-Meier method. Univariate and multivariate analyses were performed using the Cox proportional hazards model.

Results: 71 HER2 breast cancer patients were analyzed. The cut-off value of the ALC ratio was decided as 1.109. The median follow-up period was 53.1 (range: $5.1-111.5$ ) months. The high-ALC ratio group showed superior survival rates to the low-ALC ratio group ( $p=0.0242)$. The 5 -year DFS rates were $89.1 \%$ and $63.3 \%$ in the high- and low-ALC ratio group, respectively. The ALC ratio was nominated as an independent prognostic factor in multivariate Cox proportional hazards analysis $(p=0.0052)$.

Conclusion: HER2-positive breast cancer patients who showed a higher ALC ratio during trastuzumabbased neoadjuvant chemotherapy were associated to better survival.

\section{Introduction}

Neoadjuvant chemotherapy (NAC) in breast cancer was initially introduced to improve surgical outcomes in patients with locally advanced disease [1]. The use of NAC has increased the rate of breast conserving surgery and enabled treatment and response monitoring and the development of individualized strategies [2]. Treatment strategies may be tailored to cancer molecular subtypes, and NAC may improve outcomes in subgroups of patients with early-stage breast cancer [3]. Patients with human epidermal growth factor receptor-2 (HER2)-positive breast cancer and triple negative breast cancer (TNBC) have a higher chance of achieving pathological complete response ( $\mathrm{pCR}$ ) than do patients with estrogen receptor-positive breast cancer $[4,5]$.

Several studies have shown that peripheral hematologic parameters, such as the neutrophil-tolymphocyte ratio (NLR), platelet-to-lymphocyte ratio (PLR), and absolute lymphocyte count (ALC), are associated with prognosis in patients with breast cancer [6-8]. However, these studies mainly focused on pre-treatment parameters, and clinical significance of the change from pre- to post-treatment, which may reflect the host inflammatory and immune responses to NAC, has rarely been studied.

In HER2-positive breast cancer, adding trastuzumab to neoadjuvant chemotherapy has been shown to improve both the $\mathrm{PCR}$ rate and patient prognosis $[9,10]$. The mechanisms underlying the anti-tumor 
activity of trastuzumab include direct anti-proliferative effects associated with the apoptosis of tumor cells as well as the effects on the immune system via antibody-dependent cell-mediated cytotoxicity (ADCC) that involves immune effector cells. ADCC is associated with the activation of natural killer cells (NK) that express the Fc gamma receptor, which binds the Fc domain of trastuzumab [11]. ADCC activates tumor-specific cytotoxic $T$ lymphocytes [12]. During these cancer immunoediting phases, activated effector cytotoxic $T$ lymphocytes can migrate to the tumor and infiltrate its microenvironment [13]. Trafficking of T cells from the circulation to the tumor microenvironment is critical for a successful immune response against pathogens and cancer [3]. Therefore, lymphocyte induction by NAC treatment is considered to be an important indicator, but there are no reports on it. Herein, we hypothesized that patients whose peripheral lymphocytes were induced by NAC treatment with trastuzumab may have a better prognosis than do their counterparts. In addition, taxanes in combination with trastuzumab may regulate tumor microenvironment [14]; accordingly, this study also examined the impact of taxane use in TNBC.

\section{Patients And Methods}

We retrospectively identified 85 patients with stage I-III HER2-positive breast cancer and 41 patients with stage I-III TNBC treated with NAC in the Tokushima University Hospital between January 2010 and December 2019. A total of 14 patients with the following characteristics, 1) HER2-positive breast cancer treated with NAC without trastuzumab, 2) incomplete blood sample data, and 3) NAC discontinued due to adverse events were excluded. The final sample included 71 and 41 patients with HER2-positive and TNBC breast cancer, respectively. The follow-up period ended in September 2020.

\section{Neoadjuvant chemotherapy}

Patients with HER2-positive breast cancer were preoperatively treated with a trastuzumab plus taxane regimen. Many patients were also treated with an anthracycline-based combination regimen before receiving taxane. This combination regimen involved four cycles of EC (epirubicine $90 \mathrm{mg} / \mathrm{m} 2$, cyclophosphamide $600 \mathrm{mg} / \mathrm{m} 2$, q3w). Taxane regimens involved 12 cycles of weekly paclitaxel (80 $\mathrm{mg} / \mathrm{m} 2)$ or four cycles of tri-weekly docetaxel $(75 \mathrm{mg} / \mathrm{m} 2)$ doses. Some patients received four cycles of tri-weekly docetaxel $(40 \mathrm{mg} / \mathrm{m} 2)$ and $\mathrm{S}-1(40 \mathrm{mg} / \mathrm{m} 2$ orally twice per day on days $1-14)$ regimen $(\mathrm{N}-1$ Study) prior to receiving trastuzumab and taxane regimen [15]. Trastuzumab was administered either at a dose of $4 \mathrm{mg} / \mathrm{kg}$ at loading, followed by $2 \mathrm{mg} / \mathrm{kg}$ on a weekly basis, or at a dose of $8 \mathrm{mg} / \mathrm{kg}$ at loading, followed by $6 \mathrm{mg} / \mathrm{kg}$ every 3 weeks. In a few patients, pertuzumab was used in combination with trastuzumab. Pertuzumab was administered at a dose of $840 \mathrm{mg}$ at loading, followed by $420 \mathrm{mg}$ every 3 weeks. In TNBC, the NAC regimen consisted of four cycles of EC, followed by four cycles of tri-weekly docetaxel $(75 \mathrm{mg} / \mathrm{m} 2)$ or 12 cycles of weekly paclitaxel $(80 \mathrm{mg} / \mathrm{m} 2)$. Some patients received the $\mathrm{N}-1$ Study regimen.

ALC ratio and cut-off value 
In HER2-positive breast cancer patients, a routine peripheral blood test was performed, and lymphocyte count estimates were obtained before trastuzumab administration (pre-treatment ALC). Subsequently, routine blood tests were performed before the fourth cycle of trastuzumab and docetaxel regimen or before the tenth cycle of trastuzumab and paclitaxel regimen. Changes to ALC after NAC were calculated (post-treatment ALC). The ALC ratio was defined as the pre-treatment ALC/post-treatment ALC values.

In TNBC patients, the ALC ratio was calculated based on estimates obtained from routine blood tests before taxane administration (pre-treatment ALC) and those obtained before the fourth cycle of docetaxel regimen or before the tenth cycle of paclitaxel regimen.

\section{Evaluation of clinicopathological factors}

Data on clinical and pathological characteristics of patients were extracted from their medical records, including age, $\mathrm{T}, \mathrm{N}$, stage, estrogen receptor status, HER2 status, and histological therapeutic effect. Parameters such as $\mathrm{T}, \mathrm{N}$, and stage were clinically evaluated using pre-treatment imaging findings according to the Union for International Cancer Control TNM classification [16]. Estrogen receptor and HER2 status were determined using biopsy specimens obtained prior to NAC.

Estrogen receptor-positive findings were defined as nuclear staining in $\geq 10 \%$ of cancer cells. HER2 protein expression was classified into the following four groups: $0,1+, 2+$, and $3+$. Samples of patients with 2+ expression of HER2 were also tested using in situ hybridization for gene amplification. Protein and in situ hybridization were both performed following the American Society of Clinical Oncology/College of American Pathologists guidelines for HER2 testing [17]. HER2-positivity was defined as HER2 protein expression of 3+ or HER2 gene amplification. Histopathological therapeutic effects were evaluated based on whether PCR was achieved, defined as the disappearance of invasive cancer nests in breast tissue.

\section{Statistical analyses}

All statistical analyses were performed with JMP Ver.14 (SAS Institute Inc., Cary, NC, USA). The $\chi^{2}$ test was used to determine significant differences in relationships between the ALC ratio (low vs. high) and clinicopathological factors. The ROC curve analysis was performed to determine the cut-off value of the ALC ratio associated with disease recurrence. The Kaplan-Meier curves were generated to estimate disease-free survival (DFS), and the log rank test was used to compare survival between the groups. Pvalues of $<0.05$ were considered indicative of a statistically significant finding. Univariate and multivariate analyses of DFS estimates were performed using the Cox proportional hazards model. Multivariate Cox regression analysis included factors such as the ALC ratio (low vs. high), pathology findings (non-pCR vs. pCR), age (>50 vs. $\leq 50$ years), tumor size (T3-4 vs. T1-2), and nodal status (N1-3 vs. N0).

\section{Ethical statement}


The study was conducted in accordance with the Declaration of Helsinki. The institutional review board of the Tokushima University Hospital approved this study (CR 3672) and waived the informed consent requirement due to the retrospective nature of this study.

\section{Results}

Table 1 presents the clinical characteristics of HER2-positive breast cancer patients and estimates of the association between these factors and the ALC ratio. The area under the curve for the ALC ratio associated with disease recurrence in HER2-positive breast cancer was 0.631 (Fig.1). The cut-off value was determined as 1.109 , based on the receiver operating characteristic (ROC) curve analysis and Youden's index. Patients were categorized into either a high- (ALC ratio of $>1.109$ ) and low-ALC (ALC ratio of $<1.109$ ) ratio groups. The median age of patients at the start of treatment was 58 (range 35-82) years. A total of 59 and 12 patients had T1-2 and T3-4 tumor size, respectively. Moreover, 47 patients had lymph node metastasis. A total of 10,39, and 22 patients had stage I, II, and III disease, respectively. A total of 35 cases were HR positive. HER2 expression was determined as $2+$ and $3+$ in 10 and 61 cases, respectively. A total of 52 patients were preoperatively treated with $\mathrm{EC}$ followed by taxane and an antiHER2 drug (trastuzumab and/or pertuzumab). Thirteen patients received only taxane and anti-HER2 drug before surgery. Six patients received S-1 and docetaxel, followed by taxane and trastuzumab. The highALC ratio group was significantly older than the low-ALC ratio group $(p=0.0394)$. There was no significant relationship between the ALC ratio and $\mathrm{T}, \mathrm{N}$, stage, HR, HER2, or regimen.

Table 2 presents clinical characteristics of TNBC patients and estimates of the association between the ALC ratio and clinicopathological factors. The median age of patients at the start of treatment was 60 (range 32-79) years. A total of 34 and 7 cases were classed as T1-2 and T3-4 tumor size, respectively. Twenty-nine patients had lymph node metastasis. A total of 1, 31, and 9 cases were classified as clinical stage I, II, and III. Twenty-seven patients were preoperatively treated with EC followed by taxanes. S-1 and docetaxel were administered to 14 patients.

\section{$A L C$ ratio and $p C R$ rate}

Table 3 presents the relationship between ALC ratio and pCR rate. In HER2-positive breast cancer, pCR was observed in 36 patients. The pCR rate was 59.5\% (25/42) and 37.9\% (11/29) in the high- and low$A L C$ ratio groups, respectively. There was no significant relationship between the ALC ratio and the pCR rate $(p=0.0725)$. But the high-ALC ratio group tended to have a higher $p C R$ rate than the low-ALC ratio group among HER2-positive breast cancer patients.

In TNBC, pCR was observed in 16 patients. The pCR rate was $39.3 \%(11 / 28)$ and $38.5 \%(5 / 13)$ in the highand low-ALC ratio groups, respectively. There was no significant relationship between the ALC ratio and $\mathrm{pCR}$ rate $(\mathrm{p}=0.9598)$. 
The median follow-up time for HER2-positive breast cancer patients was 49.2 (range: 8.4-109.2) months. During this period, 11 (15.5\%) patients developed recurrence, and three (4.2\%) patients died due to disease recurrence. In HER2-positive breast cancer, the high-ALC ratio group showed better survival than the low-ALC group ( $p=0.0242$ ) (Fig. 2). The 5-year DFS rates were $89.1 \%$ and $63.3 \%$ in the high- and lowALC ratio groups, respectively.

In the TNBC group, 10 (24.4\%) patients developed recurrence, and four (9.8\%) patients died due to recurrence during the follow-up period. There was no significant difference in DFS between the low- and high-ALC ratio groups ( $p=0.7723$ ) (Fig. 3). The 5 -year DFS rate was $70.9 \%$ and $76.2 \%$ in the high- and lowALC ratio groups, respectively. Multivariate Cox proportional hazards analysis revealed that age and the ALC ratio were significant prognostic factors for DFS in HER2-positive breast cancer patients (hazard ratio $=8.31 ; 95 \%$ confidence interval $=1.88-36.70 ; p=0.0052)$ (Table 4$)$.

\section{Discussion}

This study aimed to examine the factors associated with DFS in patients undergoing NAC for HER2positive breast cancer or TNBC. In the present study, patients with HER2-positive breast cancer and a higher ALC ratio after NAC showed superior DFS to their counterparts with lower ALC ratio.

Several previous studies have evaluated the impact of pre-treatment inflammatory markers such as NLR and PLR on DFS and overall survival, showing that high pre-treatment NLR was associated with poor outcomes [7]. Some studies have validated the prognostic value of blood inflammatory cell counts in HER2-positive breast cancer [18]. Guo et al. performed a meta-analysis of 39 studies to evaluate the prognostic role of NLR and PLR, showing an association between NLR and overall survival in HER2positive breast cancer patients, which was of lower magnitude than that observed in patients with TNBC [19].

In addition, several studies have examined the role of lymphocytes in prognostication. Manuel et al. suggested that lymphopenia increases the risk of disease progression and worsens long-term survival [20]. However, previous studies were based on the results of a single blood test, despite the fact that baseline blood cell counts vary between individuals. In patients undergoing chemotherapy, blood cell count may change due to treatment or the use of supportive therapies such as the granulocyte-colony stimulating factor.

The present study focused on the increase in the ALC ratio during NAC to measure the immune cell response. The high-ALC ratio group was significantly older than the low-ALC ratio group. Thymus function and output may decrease with age [21], suggesting that baseline ALC values in older patients may have been lower than those in younger patients. In multivariate analysis, older patients were at a higher risk of recurrence than younger patients. Therefore, elevated ALC ratio of HER2-positive breast cancer patients can be evaluated as an independent prognostic factor. Meanwhile, there was no difference between the ALC groups in TNBC patients. 
Trastuzumab is a humanized mAb antibody against the extracellular domain of the tyrosine kinase receptor HER2. Although trastuzumab is currently used for the treatment of HER2-positive breast cancer, its mechanism of action remains unclear. Anti-tumor effects of trastuzumab might be partly mediated by the immune system, in particular, ADCC.

ADCC results from the activation of natural killer (NK) cells that express the Fc gamma receptor, which can be bound by the Fc domain of trastuzumab [11]. The binding of the mAb via the fragment antigenbinding portion to the target tumor cell confers ADCC specificity [22]. In vivo studies have shown that mice lacking the Fc gamma receptor I and III experienced reduced anti-tumor effects of certain mAbs [23], suggesting that the cytotoxic $T$ and NK cells attracted to the tumor are tumor-infiltrating lymphocytes (TILs). TIL expression represents complicated immune response to tumor cells [24]. In HER-2-positive advanced breast cancer, higher TIL levels are associated with a greater pathological complete response in patients receiving trastuzumab-based NAC [25]. These findings suggest that an increase in the ALC may represent the level of ADCC activity. Although the mechanism of prolonged DFS in patients with increased ALC remains to be elucidated, these factors may contribute to it by activating the host immune response. Previously, pCR has been implicated in breast cancer prognostication. In the present study, the ALC ratio was more strongly associated with prognosis than was PCR. This finding suggests that the ALC ratio may be a useful preoperative prognostic factor.

There are several limitations to this study. First, this was a retrospective study with a relatively small sample size in a single center. In this study, there were only seven deaths, and overall survival estimates were not examined. Second, we extracted TNBC cases that underwent NAC at the same piriod as the HER2 group, since the patient background of the TNBC group is different from HER2 group, it cannot be said that the effect of ALC ratio is necessarily limited to the HER2 group only in this study. Third, this study did not investigate the relationship between increased ALC values and TILs; it remains unclear whether TIL activity was induced in patients with elevated ALC ratios.

In conclusion, our findings suggest that the ALC ratio increases after trastuzumab-based NAC; it may be a predictor of prolonged DFS in HER2-positive patients. The ALC ratio is an index that can be easily evaluated before surgery and may be useful in clinical practice.

\section{Abbreviations}

ALC: absolute lymphocyte count, HER2: human epidermal growth factor receptor-2, NAC: neoadjuvant chemotherapy, TNBC: triple negative breast cancer, NLR: neutrophil-to-lymphocyte ratio, PLR: platelet-tolymphocyte ratio, ADCC: antibody-dependent cell-mediated cytotoxicity, DFS: disease-free survival, TIL: tumor-infiltrating lymphocyte

\section{Declarations}

Funding: Not applicable 
Conflicts of interest/Competing interests: Not applicable

Availability of data and material: Not applicable

Code availability: Not applicable

\section{Authors' contributions}

Conceptualization: Hiroaki Inoue; Methodology: Naoki Miyamoto, Hiroaki Inoue; Formal analysis and investigation: Naoki Miyamoto, Hiroaki Inoue; Investigation: Naoki Miyamoto, Tomohiro Inui, Soichiro Sasa, Mariko Aoyama; Writing - original draft preparation: Naoki Miyamoto; Writing - review and editing: Hiroaki Inoue, Kazumasa Okumura, Hiroaki Toba, Hiromitsu Takizawa, Akira Tangoku; Supervision: Akira Tangoku

Ethics approval: The study design was approved by an ethics review board.

Consent to participate: Disclosure documents pertaining to this study were published on the website at department of Thoracic Endocrine Surgery and Oncology, Tokushima University Graduate School of Biomedical Sciences. Posting disclosure documents granted a waiver of the informed consent requirement.

Consent for publication: Patients signed informed consent regarding publishing their data.

\section{References}

1. Kaufmann M, von Minckwitz G, Bear HD, Buzdar A, McGale P, Bonnefoi H, Colleoni M, Denkert C, Eiermann W, Jackesz R, Makris A, Miller W, Pierga JY, Semiglazov V, Schneeweiss A, Souchon R, Stearns V, Untch M, Loibl S (2007) Recommendations from an international expert panel on the use of neoadjuvant (primary) systemic treatment of operable breast cancer: new perspectives 2006. Ann Oncol 18:1927-1934. https://doi.org/10.1093/annonc/mdm201

2. Untch M, Konecny GE, Paepke S, von Minckwitz G (2014) Current and future role of neoadjuvant therapy for breast cancer. Breast 23:526-537. https://doi.org/10.1016/j.breast.2014.06.004

3. von Minckwitz G, Untch M, Blohmer JU, Costa SD, Eidtmann H, Fasching PA, Gerber B, Eiermann W, Hilfrich J, Huober J, Jackisch C, Kaufmann M, Konecny GE, Denkert C, Nekljudova V, Mehta K, Loibl S (2012) Definition and impact of pathologic complete response on prognosis after neoadjuvant chemotherapy in various intrinsic breast cancer subtypes. J Clin Oncol 30:1796-1804. https://doi.org/10.1200/JC0.2011.38.8595

4. Cortazar P, Zhang L, Untch M, Mehta K, Costantino JP, Wolmark N, Bonnefoi H, Cameron D, Gianni L, Valagussa P, Swain SM, Prowell T, Loibl S, Wickerham DL, Bogaerts J, Baselga J, Perou C, Blumenthal G, Blohmer J, Mamounas EP, Bergh J, Semiglazov V, Justice R, Eidtmann H, Paik S, Piccart M, Sridhara R, Fasching PA, Slaets L, Tang S, Gerber B, Geyer CE Jr, Pazdur R, Ditsch N, Rastogi P, Eiermann W, von Minckwitz G (2014) Pathological complete response and long-term 
clinical benefit in breast cancer: the CTNeoBC pooled analysis. Lancet 384:164-172. https://doi.org/10.1016/S0140-6736(13)62422-8

5. Berruti A, Amoroso V, Gallo F, Bertaglia V, Simoncini E, Pedersini R, Ferrari L, Bottini A, Bruzzi P, Sormani MP (2014) Pathologic complete response as a potential surrogate for the clinical outcome in patients with breast cancer after neoadjuvant therapy: a meta-regression of 29 randomized prospective studies. J Clin Oncol 32:3883-3891. https://doi.org/10.1200/JC0.2014.55.2836

6. Hong J, Chen X, Gao W, Zhu S, Wu J, Huang O, He J, Zhu L, Chen W, Li Y, Fei X, Lin L, Shen K (2019) A high absolute lymphocyte count predicts a poor prognosis in HER-2- positive breast cancer patients treated with trastuzumab. Cancer Manag Res 11:3371-3379.

https://doi.org/10.2147/CMAR.S187233

7. Ethier JL, Desautels D, Templeton A, Shah PS, Amir E (2017) Prognostic role of neutrophil-tolymphocyte ratio in breast cancer: a systematic review and meta-analysis. Breast Cancer Res 19:2. https://doi.org/10.1186/s13058-016-0794-1

8. Krenn-Pilko SK, Langsenlehner U, Thurner EM, Stojakovic T, Pichler M, Gerger A, Kapp KS, Langsenlehner T (2014) The elevated preoperative platelet-to-lymphocyte ratio predicts poor prognosis in breast cancer patients. Br J Cancer 110:2524-2530.

https://doi.org/10.1038/bjc.2014.163

9. Valachis A, Mauri D, Polyzos NP, Chlouverakis G, Mavroudis D, Georgoulias V (2011) Trastuzumab combined to neoadjuvant chemotherapy in patients with HER2-positive breast cancer: a systematic review and meta-analysis. Breast 20:485-490. https://doi.org/10.1016/j.breast.2011.06.009

10. Buzdar AU, Valero V, Ibrahim NK, Francis D, Broglio KR, Theriault RL, Pusztai L, Green MC, Singletary SE, Hunt KK, Sahin AA, Esteva F, Symmans WF, Ewer MS, Buchholz TA, Hortobagyi GN (2007) Neoadjuvant therapy with paclitaxel followed by 5-fluorouracil, epirubicin, and cyclophosphamide chemotherapy and concurrent trastuzumab in human epidermal growth factor receptor 2-positive operable breast cancer: an update of the initial randomized study population and data of additional patients treated with the same regimen. Clin Cancer Res 13:228-233. https://doi.org/10.1158/10780432.CCR-06-1345

11. Valabrega G, Montemurro F, Aglietta M (2007) Trastuzumab: mechanism of action, resistance and future perspectives in HER2-overexpressing breast cancer. Ann Oncol 18:977-984. https://doi.org/10.1093/annonc/mdl475

12. Andre F, Dieci MV, Dubsky P, Sotiriou C, Curigliano G, Denkert C, Loi S (2013) Molecular pathways: involvement of immune pathways in the therapeutic response and outcome in breast cancer. Clin Cancer Res 19:28-33. https://doi.org/10.1158/1078-0432.CCR-11-2701

13. Chen DS, Mellman I (2013) Oncology meets immunology: the cancer-immunity cycle. Immunity 39:1-10. https://doi.org/10.1016/j.immuni.2013.07.012

14. Zhang L, Dermawan K, Jin M, Liu R, Zheng H, Xu L, Zhang Y, Cai Y, Chu Y, Xiong S (2008) Differential impairment of regulatory $T$ cells rather than effector $T$ cells by paclitaxel-based chemotherapy. Clin Immunol 129:219-229. https://doi.org/10.1016/j.clim.2008.07.013 
15. Nakagawa $M$, Ikeuchi M, Morimoto $M$, Takechi $H$, Toba $H$, Yoshida T, Okumura K, Hino N, Nishisho A, Tangoku A (2019) Phase II study of S-1 combined with low-dose docetaxel as neoadjuvant chemotherapy for operable breast cancer patients (N-1 study). Clin Breast Cancer 19:10-16. https://doi.org/10.1016/j.clbc.2018.09.008

16. Leslie HS, Mary KG, Christian W (2009) TNM classification of malignant tumours, 7th ed. Hodoken, NJ

17. Wolff AC, Hammond MEH, Allison KH, Harvey BE, Mangu PB, Bartlett JMS, Bilous M, Ellis IO, Fitzgibbons P, Hanna W, Jenkins RB, Press MF, Spears PA, Vance GH, Viale G, McShane LM, Dowsett M (2018) Human epidermal growth factor Receptor 2 testing in breast cancer: American Society of Clinical Oncology/College of American Pathologists clinical practice guideline focused update. Arch Pathol Lab Med 142:1364-1382. https://doi.org/10.5858/arpa.2018-0902-SA

18. Tiainen S, Rilla K, Hämäläinen K, Oikari S, Auvinen P (2021) The prognostic and predictive role of the neutrophil-to-lymphocyte ratio and the monocyte-to-lymphocyte ratio in early breast cancer, especially in the HER2 + subtype. Breast Cancer Res Treat 185:63-72. https://doi.org/10.1007/s10549-020-05925-7'

19. Guo W, Lu X, Liu Q, Zhang T, Li P, Qiao W, Deng M (2019) Prognostic value of neutrophil-tolymphocyte ratio and platelet-to-lymphocyte ratio for breast cancer patients: an updated metaanalysis of 17079 individuals. Cancer Med 8:4135-4148. https://doi.org/10.1002/cam4.2281

20. Manuel M, Tredan O, Bachelot T, Clapisson G, Courtier A, Parmentier G, Rabeony T, Grives A, Perez S, Mouret JF, Perol D, Chabaud S, Ray-Coquard I, Labidi-Galy I, Heudel P, Pierga JY, Caux C, Blay JY, Pasqual N, Ménétrier-Caux C (2012) Lymphopenia combined with low TCR diversity (divpenia) predicts poor overall survival in metastatic breast cancer patients. Oncoimmunology 1:432-440. https://doi.org/10.4161/onci.19545

21. Linton PJ, Dorshkind K (2004) Age-related changes in lymphocyte development and function. Nat Immunol 5:133-139. https://doi.org/10.1038/ni1033

22. Lo Nigro CL, Macagno M, Sangiolo D, Bertolaccini L, Aglietta M, Merlano MC (2019) NK-mediated antibody-dependent cell-mediated cytotoxicity in solid tumors: biological evidence and clinical perspectives. Ann Transl Med 7:105. https://doi.org/10.21037/atm.2019.01.42

23. Eccles SA (2001) Monoclonal antibodies targeting cancer: 'magic bullets' or just the trigger? Breast Cancer Res 3:86-90. https://doi.org/10.1186/bcr276

24. Bianchini G, Gianni L (2014) The immune system and response to HER2-targeted treatment in breast cancer. Lancet Oncol 15:e58-e68. https://doi.org/10.1016/S1470-2045(13)70477-7

25. Inoue H, Horii R, Ito Y, Iwase T, Ohno S, Akiyama F (2018) Tumor-infiltrating lymphocytes affect the efficacy of trastuzumab-based treatment in human epidermal growth factor receptor 2-positive breast cancer. Breast Cancer 25:268-274. https://doi.org/10.1007/s12282-017-0822-8

\section{Tables}

Table 1 
Patients characteristics and relationships between ALC ratio and clinicopathological factors in HER2 breast cancer 


\begin{tabular}{|c|c|c|c|c|}
\hline & \multirow[t]{2}{*}{ All patients } & Low & High & \multirow[t]{2}{*}{ p-value } \\
\hline & & ALC ratio & ALC ratio & \\
\hline & $N=71$ & $N=29$ & $\mathrm{~N}=42$ & \\
\hline Median follow-up (months) & 53.1(5.1-111.5) & $53.1(11.6-111.5)$ & $53.2(5.1-91.7)$ & 0.8872 \\
\hline Median age & $58(35-82)$ & $49(35-75)$ & $59(35-82)$ & 0.0394 \\
\hline \multicolumn{5}{|l|}{$\mathrm{T}$} \\
\hline $1-2$ & 59 & 24 & 35 & 0.9494 \\
\hline $3-4$ & 12 & 5 & 7 & \\
\hline \multicolumn{5}{|l|}{ N } \\
\hline Positive & 47 & 19 & 28 & 0.9199 \\
\hline Negative & 24 & 10 & 14 & \\
\hline \multicolumn{5}{|l|}{ Stage } \\
\hline I & 10 & 6 & 4 & 0.1402 \\
\hline II & 39 & 12 & 27 & \\
\hline III & 22 & 11 & 11 & \\
\hline \multicolumn{5}{|l|}{ HR } \\
\hline Positive & 35 & 16 & 19 & 0.4102 \\
\hline negative & 36 & 13 & 23 & \\
\hline \multicolumn{5}{|l|}{ HER2 } \\
\hline $2+$ & 10 & 4 & 6 & 0.9532 \\
\hline $3+$ & 61 & 25 & 36 & \\
\hline \multicolumn{5}{|l|}{ Regimen } \\
\hline $\begin{array}{l}\text { EC followed by } \\
\text { Taxane+Tmab(+Pmab) }\end{array}$ & 52 & $19 *$ & $33^{\star \star \star}$ & 0.2465 \\
\hline Taxane+Tmab(+Pmab) & 13 & $8^{\star *}$ & 5 & \\
\hline $\begin{array}{l}\text { S-1+DTX followed by } \\
\text { Taxane+Tmab }\end{array}$ & 6 & 2 & 4 & \\
\hline
\end{tabular}


ALC Absolute lymphocyte counts, HR Hormone receptor, Tmab Trastuzumab, Pmab Pertuzumab, DTX Docetaxel

* Including 2 cases of Pmab administration

** Including 3 cases of Pmab administration

*** Including 8 cases of Pmab administration

Table 2

Patients characteristics and relationships between ALC ratio and clinicopathological factors in triple negative breast cancer 


\begin{tabular}{|c|c|c|c|c|}
\hline & All patients & $\begin{array}{l}\text { Low } \\
\text { ALC ratio }\end{array}$ & $\begin{array}{l}\text { High } \\
\text { ALC ratio }\end{array}$ & P-value \\
\hline & $\mathrm{N}=41$ & $N=13$ & $\mathrm{~N}=28$ & \\
\hline Median follow-up (months) & $51.8(7.6-122.5)$ & 76.3(7.6-122.0) & $48.1(8.1-122.5)$ & 0.0153 \\
\hline Median age & $60(32-79)$ & $59(37-79)$ & $59(32-71)$ & 0.6623 \\
\hline \multicolumn{5}{|l|}{$\mathrm{T}$} \\
\hline $1-2$ & 34 & 11 & 23 & 0.8436 \\
\hline $3-4$ & 7 & 2 & 5 & \\
\hline \multicolumn{5}{|l|}{$N$} \\
\hline Positive & 29 & 10 & 19 & 0.5474 \\
\hline Negative & 12 & 3 & 9 & \\
\hline \multicolumn{5}{|l|}{ Stage } \\
\hline I & 1 & 0 & 1 & 0.5062 \\
\hline II & 31 & 11 & 20 & \\
\hline III & 9 & 2 & 7 & \\
\hline \multicolumn{5}{|l|}{ Regimen } \\
\hline $\begin{array}{l}\text { EC followed by } \\
\text { Taxane }\end{array}$ & 27 & 5 & 22 & 0.0126 \\
\hline S-1+DTX & 14 & 8 & 6 & \\
\hline
\end{tabular}

ALC Absolute lymphocyte counts, DTX docetaxel

Table 3

Relationship between ALC ratio and $\mathrm{pCR}$ rate 


\begin{tabular}{|lllll|}
\hline & & Low & High & P-value \\
& & ALC ratio & ALC ratio & \\
\hline HER2 & & & & \\
\hline & pCR & 11 & 25 & 0.0725 \\
\hline & non-pCR & 18 & 17 & \\
\hline Triple negative & & & & \\
\hline & pCR & 5 & 11 & 0.9598 \\
\hline & non-pCR & 8 & 17 & \\
\hline
\end{tabular}

ALC Absolute lymphocyte counts, HER2 Human epidermal growth factor receptor-2, $p C R$ pathological complete response

Table 4

Univariate and multivariate analysis of the correlation between disease free survival and clinicopathological factors in HER2 positive breast cancer

\begin{tabular}{|lllll|}
\hline & Univariate & \multicolumn{3}{l|}{ Multivariate } \\
\hline & RR(95\%Cl) & P-value & RR(95\%Cl $)$ & P-value \\
\hline ALC ratio low vs high & $4.09(1.08-15.42)$ & 0.0377 & $8.31(1.88-36.70)$ & 0.0052 \\
\hline non-pCR vs pCR & $3.11(0.82-11.75)$ & 0.094 & $2.23(0.54-9.18)$ & 0.2650 \\
\hline Age 50< vs <50 & $2.40(0.52-11.20)$ & 0.2249 & $8.18(1.53-43.86)$ & 0.0141 \\
\hline T3-4 vs T1-2 & $1.77(0.47-6.70)$ & 0.4250 & & \\
\hline N positive vs negative & $4.00(0.51-31.28)$ & 0.1122 & $5.52(0.68-44.78)$ & 0.1095 \\
\hline HR positive vs negative & $1.89(0.55-6.45)$ & 0.3022 & & \\
\hline
\end{tabular}

$R R$ Risk Ratio, $C /$ confidence interval, $A L C$ Absolute lymphocyte count, $p C R$ pathological Complete Response, $H R$ Hormone receptor

\section{Figures}




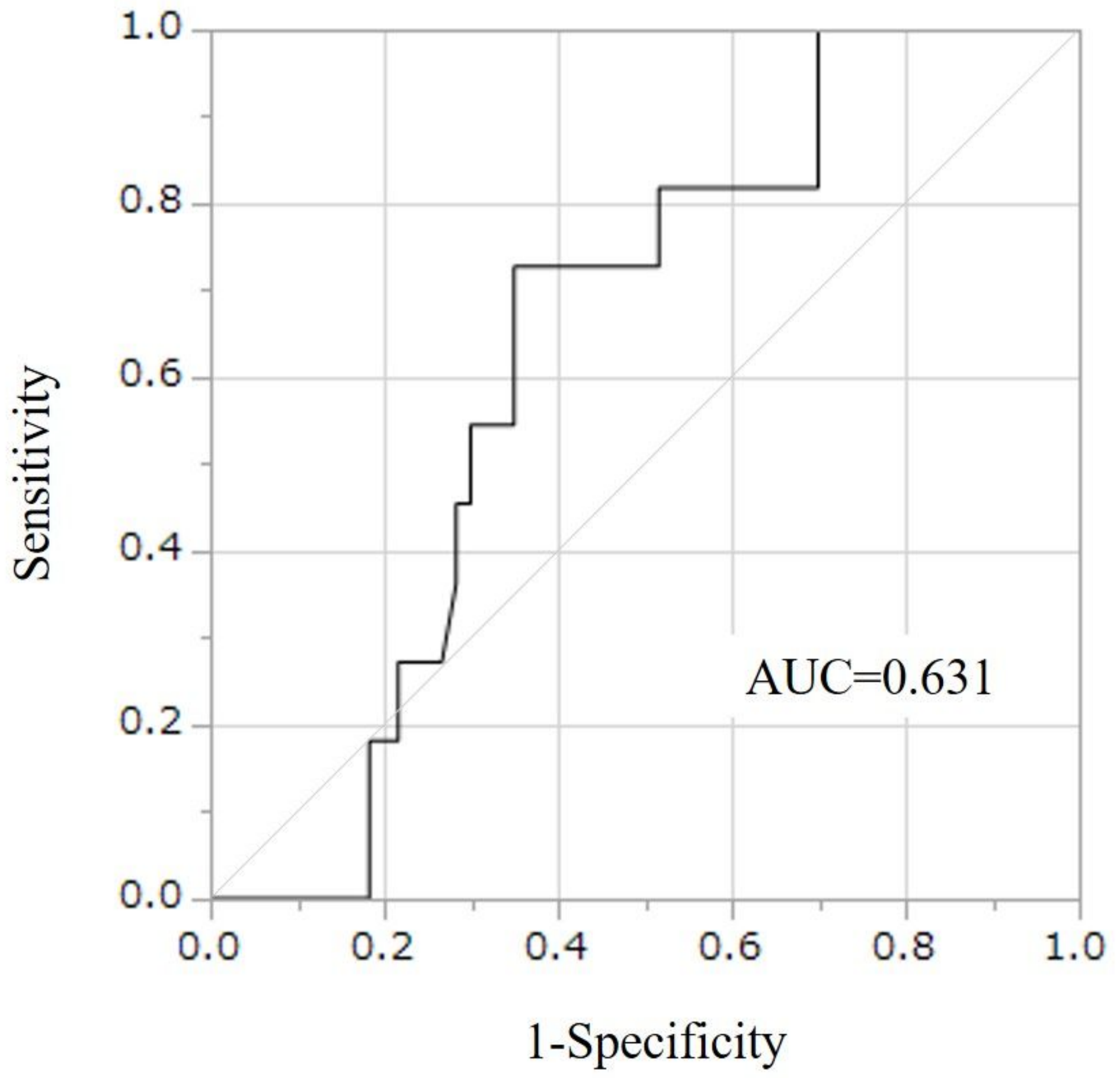

Figure 1

The cut-off value of ALC ratio in HER2+ breast cancer Area under the curve value for the absolute lymphocyte count (ALC) ratio with disease recurrence in HER2-positive breast cancer patients was 0.631. The cut-off value was determined as 1.109 with receiver operating characteristic curves and Youden's index 


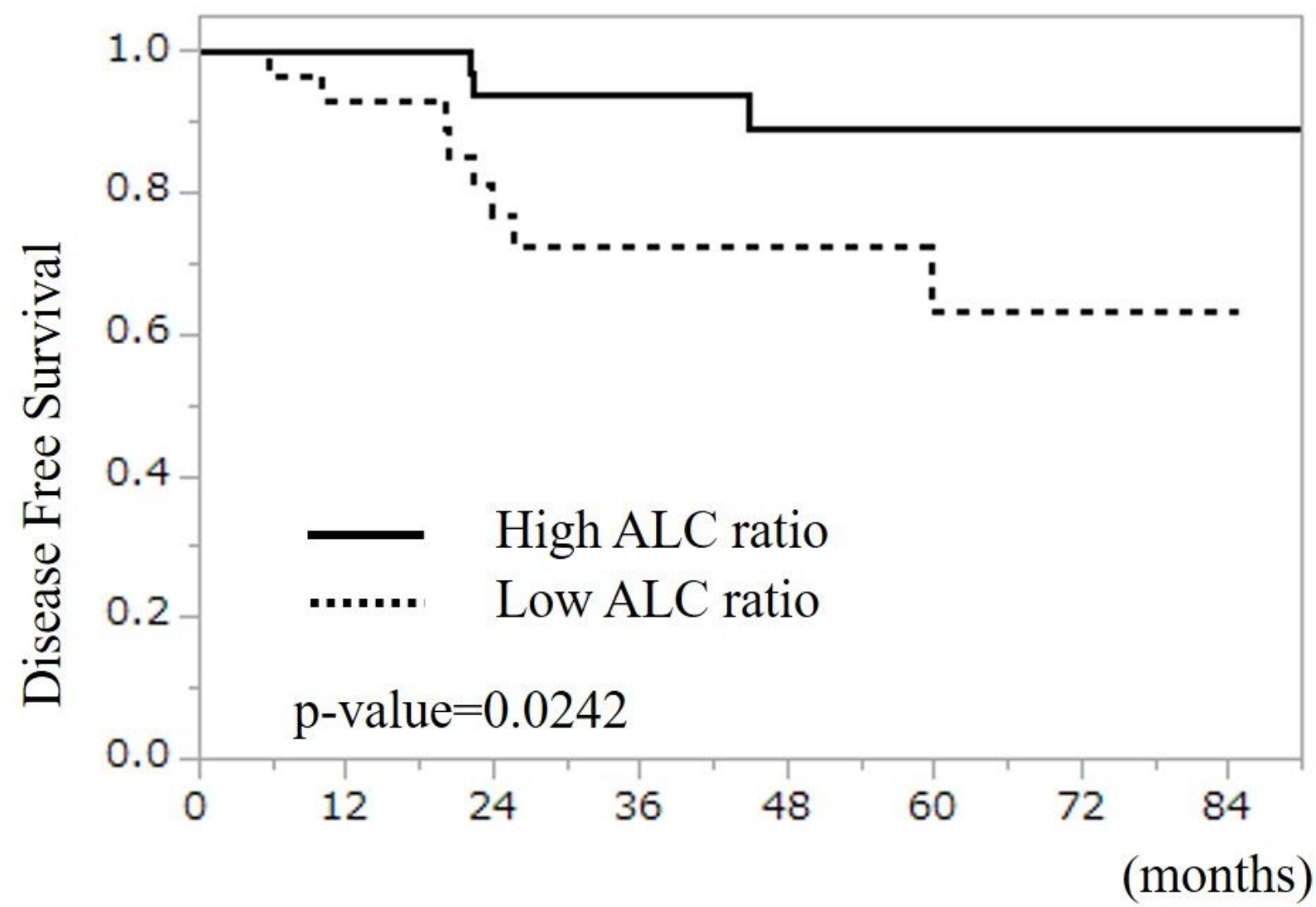

Figure 2

The 5-year disease-free survival rate in HER2+ breast cancer In HER2 positive breast cancer patients, the 5 -year disease-free rate was $89.1 \%$ and $63.3 \%$ in the high- and low-ALC ratio groups, respectively $(p=0.0242)$ 


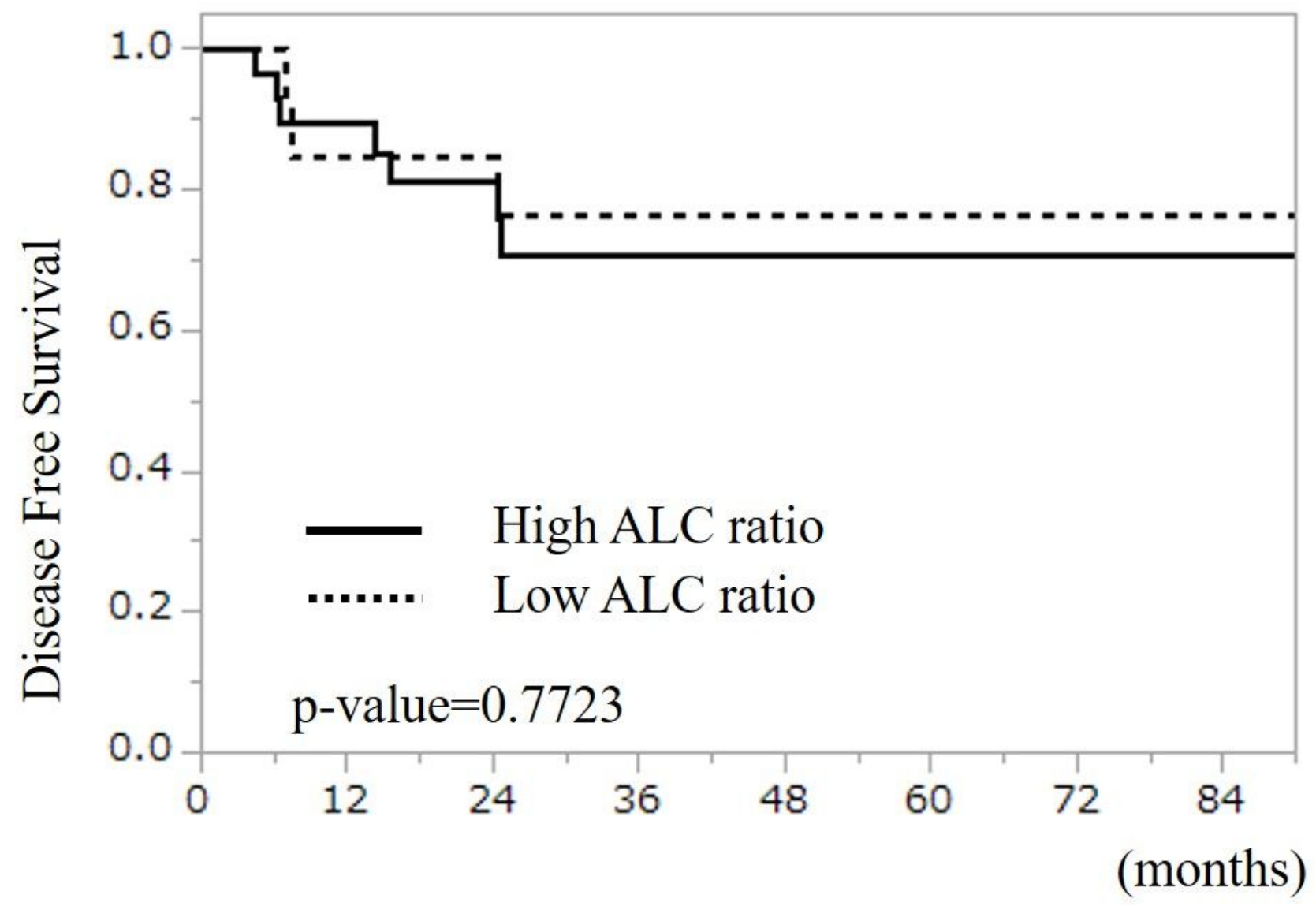

Figure 3

The 5-year disease-free survival rate in triple negative breast cancer In triple negative breast cancer, the 5year disease-free rate was $70.9 \%$ and $76.2 \%$ in high- in low-ALC ratio groups, respectively $(p=0.7723)$ 